\title{
Fungal metagenome of Chernevaya Taiga soils: taxonomic composition, differential abundance and factors related to plant gigantism
}

\author{
Rayko M. ${ }^{1 *}$, Sokornova S. ${ }^{2}$, Lapidus A. ${ }^{1}$ \\ ${ }^{1}$ Center for Algorithmic Biotechnology St. Petersburg State University, St. Petersburg, Russia \\ ${ }^{2}$ All-Russian Institute of Plant Protection, Saint-Petersburg, Russia \\ * email:m.rayko@spbu.ru
}

Chernevaya taiga of Western Siberia is a unique complex ecosystem, distinguished by extremely high vegetation, the reasons for which are poorly understood.

In this study we explored the fungal diversity of the chernevaya taiga soils in Novosibirsk and Tomsk regions in comparison with other soil types as fungal component (namely, mycorrhizal fungi) can serve as a one of the factors of high soil productivity.

Fungal components of soil biome of chernevaya taiga and control regions were investigated using ITS rRNA sequencing. We compared soil samples at two key locations in the chernevaya taiga of Salair and in the grass pine forest on the ancient terraces of the Ob River, collected in summer and fall 2019 and spring 2020. The taxonomic analysis revealed predominant fungal phyla in chernevaya taiga regions. The results demonstrate that the fungi of the chrenevaya taiga regions have higher species diversity (Faith's PD) vs control soils, and the diversity is due more to the sampling sites rather than to the seasons (Bray-Curtis distance). We studied most differentially abundant taxa between soil types, and annotated taxa by ecological guilds and trophic types. The most differentially abundant taxa in summer and fall chernevaya taiga samples belong to the phylum Glomeromycota - arbuscular mycorrhizal symbiotrophs, which are known to establish symbiotic relationships and enhance the plan growth. Also, several OTUs were assigned to novel genera in the Glomeraceae and Claroideoglomeraceae family (absent in UNITE fungal ITS database). These potential novel genera deserve further study because arbuscular mycorrhizal fungi usually can not be cultivated. Our findings add potential explanation on the high productivity and plant gigantism in chernevaya taiga, and expand our knowledge of fungal biodiversity.

Acknowledgements: The reported study was funded by Russian Scientific Foundation (grant ID 19-16-00049). 